\title{
When are we going to celebrate the centenary of the discovery of efficient treatment for congenital toxoplasmosis?
}

\author{
F Peyron \\ Laboratoire de Parasitologie et Pathologie Exotique, Hôpital de la Croix Rousse, Cedex 04, 69317 Lyon, France
}

\begin{abstract}
In 2008, we have celebrated the centenary of the discovery of Toxoplasma gondii. Although this ubiquitous protozoan can generate devastating damage in foetuses and newborns, its treatment is the only field in which we have made little progress, despite a huge body of research, and has not yet been validated. Pregnant women who seroconvert are generally given spiramycine in order to reduce the risk of vertical transmission. However, to date, we have no evidence of the efficacy of this treatment because no randomized controlled trials have as yet been conducted. When foetal contamination is demonstrated, pyrimethamine, in association with sulfadoxine or sulfadiazine, is normally prescribed, but the effectiveness of this treatment remains to be shown. With regard to postnatal treatment, opinions vary considerably in terms of drugs, regimens and length of therapy. Similarly, we do not have clear evidence to support routine antibiotic treatment of acute ocular toxoplasmosis. We must be aware that pregnant women and newborns are currently being given empirically potentially toxic drugs that have no proven benefit. We must make progress in this field through well-designed collaborative studies and by drawing the attention of policy makers to this disastrous and unsustainable situation.
\end{abstract}

Key words: congenital toxoplasmosis - treatment efficacy

In September 2008, scientists and clinicians from all over the world gathered in Brazil to celebrate the centenary of the discovery of Toxoplasma gondii. As stated by the congress organisers, this ubiquitous protozoan, which can infect humans and almost all warm-blooded animals, can generate devastating damage in fetuses, newborns and immunocompromised patients.

When it was discovered simultaneously in Tunisia and Brazil, T. gondii was not given great clinical importance, but instead, its taxonomic classification was the centre of passionate discussions. Only in 1912 Splendore did suggest, in a congress held in Paris, that "we should not be surprised if this disease would be observed in humans in the future". It was in 1923 that Janku, an ophthalmologist in Prague, reported the first case of chorioretinitis in a child. Parasites were found in the eyes and he called them "sporozoa". Wolf and Cowan, in 1938, identified Toxoplasma as the cause of neonatal encephalitis and published the first report of congenital transmission of T. gondii. In 1941, Sabin described a triad of signs (retinochoroiditis, hydrocephalus and cerebral calcification) in an infant with congenital toxoplasmosis. More recently, in the 1980s, life-threatening opportunistic infections with $T$. gondii emerged in immunocompromised patients, especially those with AIDS. In the foetus, infection can cause a wide spectrum of consequences that range from severe neurological abnormalities to subclinical presentation at birth that is marked by the risk of late onset of ocular lesions (Wallon et al. 2004).

Corresponding author: francois.peyron@chu-lyon.fr

Received 27 November 2008

Accepted 3 December 2008
Until now, $T$. gondii has elicited a huge body of research in the fields of biology, immunology and genetics, the most recent results of which are being presented during this meeting. Surprisingly, the only field in which we have made little progress is the treatment of the congenital infection. This situation is rather surprising given the following: (i) medically, congenital infection is a real burden that is certainly underestimated in terms of DALY (Havelaar et al. 2007). Some countries, such as Austria and France, have implemented a prenatal screening programme and other European countries have issued recommendations to screen non-immune pregnant women. In Europe, 28 countries recently declared that they had a system for the surveillance of congenital toxoplasmosis that could be used either routinely or only to identify symptomatic cases (Benard et al. 2008): (ii) active and well-tolerated drugs are available for control of the infection as demonstrated by the prevention and treatment of the disease in immunocompromised patients (Montoya \& Liesenfeld 2004). The emergence of opportunistic infections in AIDS patients has markedly stimulated the search for new active compounds.

With respect to the treatment of congenital toxoplasmosis, two different regimens are available: ante or postnatal treatment. The first aim of antenatal treatment is to prevent foetal infection. Therefore, prompt treatment should be given to women who seroconvert during pregnancy. After that, in cases where foetal infection is demonstrated, a more potent antiparasitic treatment is given to reduce the severity of the disease by limiting the multiplication of Toxoplasma.

During the postnatal period, infected newborns are usually treated to prevent the occurrence of chorioretinitis. When these lesions appear later in life or in the case of recurrence, similar treatment is generally given. 
With regard to the prevention of foetal infection, it is generally accepted that spiramycine should be given immediately to pregnant women who seroconvert in order to reduce the risk of vertical transmission. To date, we have no evidence of the efficacy of spriramycine because no randomised controlled trials have been conducted (Wallon et al. 1999). Currently, there is no consensus method for monitoring maternal infection. Findings in published studies have cast doubt on the effectiveness of antenatal treatment in preventing mother-to-child transmission (Gilbert et al. 2001), probably because the treatment begins too late. Another European study was unable to demonstrate a beneficial effect of antenatal treatment in lowering congenital infection, but could not exclude a clinical effect (Gilbert \& Gras 2003). Recently, a meta-analysis of data from individual patients reported weak evidence for an association between early treatment and reduced risk of congenital toxoplasmosis (Thiebaut et al. 2007). The conclusion of the authors was that observational studies would not be useful and only large randomised trials would provide valid evidence of a potential benefit of prenatal treatment.

With regard to secondary prevention, uncertainty remains regarding its efficacy in reducing the clinical manifestations of the disease. Ocular manifestations have been reported in as many as $80 \%$ of congenitally infected patients who were not given antenatal treatment. These results should be interpreted cautiously, as they might be flawed by the enrolment of only symptomatic cases. Usually, when foetal contamination is demonstrated, spiramycine is no longer administered and potent antiparasitic treatment, mainly pyrimethamine in combination with sulfadoxine or sulfadiazine, is prescribed. Whether or not this treatment is efficient remains to be demonstrated. Foulon et al. (1999) found that antenatal treatment significantly reduced sequelae but a control group was not randomly selected and follow-up was for one year only. In a 19-month follow-up, Holfeld found that pyrimethamine plus sulfonamide was effective in reducing the severity of the disease in comparison with a group of historical controls (Holfeld et al. 1989). We demonstrated that a delay of more than eight weeks between maternal seroconversion and treatment onset was associated with an increased risk of retinochorioditis during the first two years of life in infected infants (Kieffer et al. 2008).

Regarding postnatal treatment, opinions vary considerably in terms of drugs, regimens and length of treatment. This demonstrates that none prevails in terms of efficacy. In most countries, an infected newborn is treated for an average of one year. However, the compounds used in the regimen vary considerably between different centres. In most publications, postnatal treatment is usually compared with historical studies that reported poor outcomes for untreated patients. It should be born in mind that, at the time of some of these studies, antenatal diagnosis and ultrasound were not available. Mcleod et al. (2006) reported the results of a longitudinal study and found that infants who were treated for one year after birth with pyrimethamine and sulfadiazine had better outcomes that those of children who were not treated or who were treated for one month. Recently, the same team reported on a cohort of congenitally infected children who were not treated during the first year of life. The rate of eye lesions was higher than that in another cohort of children treated during the first year of life. A possible explanation for this difference is that rapid onset of treatment after birth reduces the occurrence of eye lesions (Phan et al. 2008).

Ocular lesions are the most common manifestation of congenital toxoplasmosis. New lesions can appear unexpectedly throughout life and scarred foci can be reactivated in an unpredictable way. Some periods have been reported to be at risk for recurrence such as puberty (not demonstrated to date in our cohort) or pregnancy (Garweg et al. 2005). Again, opinions about this situation differ and controversy remains about the efficacy of treatment. It is commonly accepted that, when observed for the first time, healed lesions should not be treated. As ocular toxoplasmosis is a self-limiting disease, some clinicians will not treat small peripheral lesions. Active lesions, especially those that are vision-threatening due to their localization in the retina, are usually treated with a combination of dihydrofolate inhibitors, sulfonamides and steroids. In a review, Holland and Lewis (2002) reported the use of nine drugs and 24 different regimens for the treatment of recurrent chorioretinitis. In an evidence-based systematic review, Stanford et al. (2003) investigated the effectiveness of systemic antibiotic treatment. Only three studies were randomised controlled trials and none gave clear evidence to support routine antibiotic treatment of acute ocular toxoplasmosis.

This lack of evidence results in conflicting opinions that increase the anxiety that is already present in pregnant women and doctors regarding the occurrence of maternal toxoplasmosis. Opinions also vary considerably among different countries. Even in France, despite a surveillance programme that has been on-going for more than 20 years, treatments differ among centres (Binquet et al. 2004). We must be aware that, at a time of evidence-based medicine, pregnant women and newborns are currently being given potentially toxic drugs that have no proven benefit.

This situation is ethically unacceptable. This lack of knowledge is a result of the difficulty in carrying out well-designed studies to assess the efficacy of treatment during either the ante or postnatal period.

The major limitations in conducting such studies are the low incidence of the disease and the necessity to stratify subjects according to the stage of pregnancy at maternal seroconversion. When evaluating the efficacy of ante or postnatal treatment on sequelae, difficulties are encountered regarding an appropriate follow-up. Indeed, congenital toxoplasmosis should be considered a chronic ophthalmological disease and we have demonstrated that a 10 -year follow-up is necessary for a precise evaluation of the occurrence of retinal lesions (Wallon et al. 2004). According to our clinical experience, the need to follow-up with apparently healthy infants is difficult to explain to parents, especially to mothers who want to erase from their mind painful memories and heavy feelings of guilt. The most important point in addressing the outcome of congenital toxoplasmosis is to evaluate the 
patient's quality of life. Indeed, just comparing the number of retinal scars, the impact of which on sight varies according to their localization in the retina, will not answer the parents' question: how will our child cope later in life? The same is true for cerebral calcifications, which are generally not clinically patent.

We must overcome these limitations through collaborative studies and by drawing the attention of policy makers to this disastrous and unsustainable situation. Pharmacokinetic studies must be undertaken, since information on drug metabolism in pregnant women is scarce and, in children, drug levels have also been reported to exhibit considerable interindividual variability (Corvaisier et al. 2004). Drugs conditioned to enable adaptation of the dose to gains in body weight in infants must be made available. Moreover, research should also focus on drugs that act on the parasitic cyst, as their rupture can release viable organisms.

The current question is: what should we do now until precise information on the efficacy of treatment becomes available?

Although we should tell parents that the benefits of ante and postnatal treatment have not been demonstrated, we must bear in mind that, in this emotionally difficult situation, parents are desperately seeking advice and guidelines. Given the lack of evidence for and against treatment, we should give treatment the benefit of the doubt. Recommended drugs should therefore be prescribed after the parents have been given honest and clearly understandable information. Moreover, it is urgent that a massive information campaign be implemented among young women of child-bearing age, emphasizing the importance of primary prevention with regard to eating habits. The efficacy of such a programme in preventing maternal infection has not been clearly demonstrated, but there is suggestive evidence that health education might reduce the incidence of congenital toxoplasmosis. We have to be aware that even if doctors do not know how to treat toxoplasmosis, women are more and more frequently aware that the disease exists. Through the internet, they now have access to uncontrolled and incorrect and alarmist information. Therefore, they are prone to have serology performed at any time during pregnancy, even when no surveillance programme has been implemented in their country. When serological results are ambiguous or demonstrate acute infection, a lack of appropriate information can lead to undue termination of pregnancy. It is our duty to give them the possibility of an interview with a qualified person who will answer their questions. After many years of experience in an outpatient department in a referral centre for toxoplasmosis, I am convinced that the biggest danger to the foetus is not the parasite, but the mothers' anxiety. During interviews with infected mother, some crucial information should be therefore be provided: thanks to the dramatic progress in antenatal diagnosis (amniocentesis and ultrasound), foetal infection and abnormalities can now be detected in utero; the overall risk of vertical transmission with maternal seroconversion monitored before birth is $26 \%$; among infected children, $33 \%$ have at least one retinal lesion and none of them present bi- lateral impairment (Wallon et al. 2004); in a cohort of congenitally infected patients over 18 years of age, the majority are doing well (unpublished observations).

In 2008 we celebrated the centenary of the discovery of a parasite, the most severe clinical manifestations of which remain untreatable to date. In 1968, Garin et al. (1968) reported that the level of spiramycine achieved in the placenta after treatment was able to eradicate the parasite. To our knowledge, that was the first publication on drug prevention of foetal infection.

Why not consider celebrating in 2068 , the centenary of the first publication on the prevention of vertical transmission and evaluating the progress made during this period? We will not be attending that meeting, but the message we could pass on to our young fellows is: $T$. gondii might be a dream model for scientists, but toxoplasmosis is certainly a nightmare for mothers-to-be.

Therefore, do not stop clinical research.

\section{REFERENCES}

Benard A, Petersen E, Salamon R, Chene G, Gilbert R, Salmi L 2008. Survey of European programmes for the epidemiological surveillance of congenital toxoplasmosis. Euro Surveill 13: 6.

Binquet C, Wallon M, Metral P, Gadreau M, Quantin C, Peyron F 2004. Toxoplasmosis seroconversion in pregnant women. The differing attitudes in France. Presse Med 33: 775-779.

Corvaisier S, Charpiat B, Mounier C, Wallon M, Leboucher G, A1 Kurdi M, Chaulet J, Peyron F 2004. Population pharmacokinetics of pyrimethamine and sulfadoxine in children treated for congenital toxoplasmosis. Antimicrob Agents Chemother 48: 3794-3800.

Foulon W, Villena I, Stray-Pedersen B, Decoster A, Lappalainen M, Pinon J, Jenum P, Hedman K, Naessens A 1999. Treatment of toxoplasmosis during pregnancy: a multicenter study of impact on fetal transmission and children's sequelae at age 1 year. $A m J$ Obstet Gynecol 180: 410-415.

Garin J, Pellerat M, Paillard J, Woerhle B, Hezez R 1968. Bases théoriques de la prévention par la spiramycine de la toxoplasmose congénitale chez la femme enceinte. Presse Med 76: 2268-2271.

Garweg J, Scherrer J, Wallon M, Kodjikian L, Peyron F 2005. Reactivation of ocular toxoplasmosis during pregnancy. BJOG 112: 241-242.

Gilbert R, Gras L 2003. Effect of timing and type of treatment on the risk of mother to child transmission of Toxoplasma gondii. BJOG 110: $112-120$

Gilbert R, Gras L, Wallon M, Peyron F, Ades A, Dun D 2001. Effect of prenatal treatment on mother to child transmission of Toxoplasma gondii: retrospective cohort study of 554 mother-child pairs in Lyon, France. Int J Epidemiol 30: 1303-1308.

Havelaar A , Kemmeren J, Kortbeek L 2007. Disease burden of congenital toxoplasmosis. Clin Infect Dis 44: 1467-1474.

Hohlfeld P, Daffos F, Thulliez P, Aufrant C, Couvreur J, MacAleese J, Descombey D, Forestier F 1989. Fetal toxoplasmosis: outcome of pregnancy and infant follow-up after in utero treatment. J Pediatr 115: 765-769.

Holland G, Lewis K 2002. An update on current practices in the management of ocular toxoplasmosis. Am J Ophthalmol 134: 102-114.

Janku J 1923. Pathogenesa a pathologicka anatomie tak nazveneho vrozeneho kolobomu zlute skvrny v oku normalne velikem a mickrophtalmickem s nalezem parasitu v sitnici. Cas Lek Ces 62: 1021-1027. 
Kieffer F, Wallon M, Garcia P, Thulliez P, Peyron F, Franck J 2008. Risk factors for retinochoroiditis during the first 2 years of life in infants with treated congenital toxoplasmosis. Pediatr Infect Dis J 27: 27-32.

McLeod R, Boyer K, Karrison T, Kasza K, Swisher C, Roizen N, Jalbrzikowski J, Remington J, Heydemann P, Noble A, Mets, Holfels E, Withers S, Latkany P, Meier P 2006. Outcome of treatment for congenital toxoplasmosis, 1981-2004: the National Collaborative Chicago-Based, Congenital Toxoplasmosis Study. Clin Infect Dis 42: 1383-1394.

Montoya JG, Liesenfeld O 2004 Toxoplasmosis. Lancet 363: 1965-1976.

Phan L, Kasza K, Jalbrzikowski J, Noble A, Latkany P, Kuo A, Mieler W, Meyers S, Rabiah P, Boyer K, Swisher C, Mets M, Roizen N, Cezar S, Sautter M, Remington J, Meier P, McLeod R 2008. Longitudinal study of new eye lesions in children with toxoplasmosis who were not treated during the first year of life. Am J Ophthalmol 146: 375-384.
Sabin AB 1941. Toxoplasmic encephalitis in children, JAMA 116: 807-714.

Stanford M, See S, Jones L, Gilbert R 2003. Antibiotics for toxoplasmic retinochoroiditis: an evidence-based systematic review. Ophthalmology 110: 926-931.

Thiebaut R, Leproust S, Chene G, Gilbert G 2007. Effectiveness of prenatal treatment for congenital toxoplasmosis: a meta-analysis of individual patients' data. Lancet 369: 115-122.

Wallon M, Kodjikian L, Binquet C, Garweg J, Fleury J, Quantin C, Peyron F 2004. Long-term ocular prognosis in 327 children with congenital toxoplasmosis. Pediatrics 113: 1567-1572.

Wallon M, Liou C, Garner P, Peyron F 1999. Congenital toxoplasmosis: systematic review of evidence of efficacy of treatment in pregnancy. BMJ 318: 1511-1514.

Wol A, Cowen D 1938. Granulomatous encephalomyelitis due to a protozoan (Toxoplasma or Encephalitozoon): II. Identification of a case from the literature. Bull Neurol Inst NY 7: 266-283. 